\title{
Relationship between DNA Methylation in the 5' CpG Island of the SLC47A1 (Multidrug and Toxin Extrusion Protein MATE1) Gene and Interindividual Variability in MATE1 Expression in the Human Liver $\mathbf{s}$
}

\author{
Toshihiro Tanaka, Takeshi Hirota, and Ichiro leiri \\ Division of Clinical Pharmacy, Department of Clinical Pharmacokinetics, Graduate School of Pharmaceutical Sciences, Kyushu \\ University, Fukuoka, Japan
}

Received June 1, 2017; accepted October 18, 2017

\begin{abstract}
Multidrug and toxin extrusion protein 1 (MATE1), which is encoded by solute carrier 47A1 (SLC47A1), mediates the excretion of organic cations into bile and urine. Some genetic variants in human MATE1 altered its transport function in in vitro experiments; however, differences in the pharmacokinetics of substrate drugs cannot be explained by genetic variations in humans. In this study, we investigated whether DNA methylation was involved in interindividual variability in MATE1 expression in the human liver. Approximately 20 -fold interindividual variability in MATE1 mRNA expression levels was observed in liver samples and mRNA expression levels negatively correlated with
\end{abstract}

methylation levels of the $\mathrm{CpG}$ island in the $27 \mathrm{~kb}$ upstream of SLC47A1. DNA demethylation by treatment with 5-aza-2'deoxycytidine increased MATE1 mRNA expression in MATE1negative cell lines. The luciferase reporter assay showed that the $\mathrm{CpG}$ island increased the transcriptional activity of the SLC47A1 promoter. MATE1 mRNA expression levels were significantly lower in $\mathrm{CpG}$ island knockout HepG2 cells than in control cells. These results suggest that the $5^{\prime} \mathrm{CpG}$ island of SLC47A1 acts as an enhancer for SLC47A1, and DNA methylation in the CpG island plays an important role in interindividual differences in hepatic MATE1 expression.

\section{Introduction}

Interindividual variability in drug responses is a major clinical issue for effective drug treatment. Since drug pharmacokinetics are related to the functions of drug transporters, variations in drug transporter gene expression have been identified as one of the causes of this issue. Previous studies reported that genetic variations in drug transporters altered the responses of some clinical drugs (Niemi et al., 2005; Yamasaki et al., 2008); however, the main reasons for interindividual variability in drug responses currently remain unclear. The molecular mechanisms regulating gene expression need to be elucidated in more detail to resolve this issue.

Multidrug and toxin extrusion protein 1 (MATE1) was identified as a mammalian ortholog of the bacterial MATE family in 2005 (Otsuka et al., 2005) and has been demonstrated to function as an $\mathrm{H}^{+}$/organic cation antiporter. Human MATE1 is strongly expressed in the kidney and liver and is located in the luminal membrane (Otsuka et al., 2005; Masuda

This work was supported by the Japan Society for the Promotion of Science [KAKENHI Grant-in-Aid for Scientific Research (C) 17H04103].

https://doi.org/10.1124/mol.117.109553.

S This article has supplemental material available at molpharm. aspetjournals.org. et al., 2006). Various drugs, including metformin, cimetidine, and topotecan, have been identified as substrates of MATE1. Significantly high hepatic concentrations of metformin have been reported in Mate1 knockout mice (Toyama et al., 2012). This finding suggests that hepatic MATE1 expression levels affect the pharmacokinetics of substrate drugs. Since some genetic variants in human MATE1 have been shown to alter its transport function in in vitro experiments, several clinical studies were performed to assess the relationship between solute carrier 47A1 (SLC47A1) genotypes and the pharmacokinetics of metformin (Tzvetkov et al., 2009; Christensen et al., 2011; Stocker et al., 2013). However, most studies demonstrated that these variants did not affect the pharmacokinetic parameters of metformin. Therefore, other factors may contribute to interindividual variability in the function of MATE1.

In addition to genetic variations, epigenetic mechanisms are regarded as important factors that alter gene expression. Epigenetics is defined as molecular factors and processes around DNA that regulate gene expression independent of DNA sequences. In epigenetic mechanisms, DNA methylation is one of the most studied epigenetic marks. DNA methylation occurs at the 5-carbon position of cytosine residues located in dinucleotide $\mathrm{CpG}$ sites and is associated with the regulation of

ABBREVIATIONS: 3C, chromosome conformation capture; 5-aza-dC, 5-aza-2'-deoxycytidine; cDNA, complementary DNA; CGI, CpG island; CRISPR, clustered regularly interspaced short palindromic repeats; DNMT, DNA methyltransferase; GAPDH, glyceraldehyde 3-phosphate dehydrogenase; PCR, polymerase chain reaction; $\mathrm{PPCR}$, quantitative polymerase chain reaction; RT, reverse transcription; sgRNA, single guide RNA; TSS, transcriptional start site. 
embryonic development, transcription, chromatin structure, $\mathrm{X}$-chromosome inactivation, genome imprinting, and chromosome stability (Bird, 2002). In mammals, regions of high $\mathrm{CpG}$ density, called CpG islands (CGIs), have been observed in the promoter regions of $60 \%-70 \%$ of genes (Saxonov et al., 2006). Although most CGIs at promoter regions are unmethylated, the methylation of CpG-rich promoters is essentially inactive (Deaton and Bird, 2011).

The role of DNA methylation in gene transcription, including that of drug transporters in cancer cells, has been studied in detail. For example, previous studies reported that methylation levels of the promoter of ATPbinding cassette, subfamily G, member 2 (which codes the breast cancer resistance protein) inversely correlated with breast cancer resistance protein mRNA expression levels in some cancer cells (To et al., 2006; Bram et al., 2009). Since cancer-specific changes in DNA methylation have been observed in a number of cancer-related genes, researchers have focused on DNA methylation-based biomarkers to diagnose various tumor entities and sensitivity (Warton and Samimi, 2015; Moon et al., 2016). The contributions of DNA methylation to interindividual variability in expression in normal tissue have also been evaluated (Yasar et al., 2013; Wu et al., 2015). These findings suggest that individualized drug therapy is possible using DNA methylation-based biomarkers. However, there are currently no reports on the analysis of DNA methylation in interindividual differences in MATE1 expression.

In this study, we examined whether DNA methylation was involved in interindividual variability in MATE1 expression in the human liver. Since the methylated region located in the $30 \mathrm{~kb}$ upstream of the transcriptional start site (TSS) of genes regulates gene expression (Yoon et al., 2002, 2005), we analyzed the methylation levels of CGIs within the $30 \mathrm{~kb}$ upstream of SLC47A1. We also investigated the effects of CGI on MATE1 expression and the mechanisms by which CGIs interact with the $S L C 47 A 1$ gene locus.

\section{Materials and Methods}

Tissue Samples. Twenty-one human liver samples were obtained from Caucasian donors at the National Disease Research Interchange (Philadelphia, PA). All liver samples were excised from each individual liver. The characteristics of the liver samples are shown in Table 1. Tissues were frozen in liquid nitrogen immediately after sampling and stored at $-80^{\circ} \mathrm{C}$. This study was approved by the Ethical Board of the Faculty of Medicine of Kyushu University.

Cell Culture. The HepG2 cell line was obtained from the RIKEN Cell Bank (Tsukuba, Japan). The HEK293 and LS174T cell lines were obtained from Dainippon Pharmaceuticals (Osaka, Japan). The $\mathrm{HaCaT}$ cell line was obtained from Cell Lines Service (Heidelberg, Germany). All cell lines were grown at $37^{\circ} \mathrm{C}$ in an incubator with $5 \%$ $\mathrm{CO}_{2}$. The HepG2, HEK293, and HaCaT cell lines were maintained in Dulbecco's modified Eagle's medium (Sigma-Aldrich, St. Louis, MO). The LS174T cell line was maintained in Eagle's minimum essential medium (Wako, Osaka, Japan). All media were supplemented with $10 \%$ fetal bovine serum.

TABLE 1

Characteristics of human liver tissues

\begin{tabular}{cc}
\hline Characteristic & Value \\
\hline Sex (male/female) & $16 / 5$ \\
Age range (years) & $17-70$ \\
\hline
\end{tabular}

Nucleic Acid Extraction and Complementary DNA Synthesis. Total RNA and genomic DNA from human liver tissues and cell lines were isolated with the RNeasy Plus Mini Kit (QIAGEN, Valencia, CA) or the NucleoSpin Tissue Kit (TaKaRa, Tokyo, Japan). First-strand complementary DNA (cDNA) synthesis was performed using SuperScript II (Invitrogen, Carlsbad, CA) and a random primer (Promega, Madison, WI). Reverse transcription (RT) reactions were always performed in the presence or absence of reverse transcriptase to ensure that genomic DNA did not contaminate the subsequent polymerase chain reaction (PCR).

Quantification of MATE1 mRNA Expression in the Human Liver. Real-time PCR was performed with SYBR Premix Ex Taq (TakaRa). The specificity of the real-time PCR product was proven by a dissociation curve analysis. To compare gene expression levels among different samples, we normalized values using an endogenous reference gene, glyceraldehyde 3-phosphate dehydrogenase (GAPDH). Real-time PCR primers were applied to the quantitative analysis of mRNA expression (Supplemental Table 1) using the StepOnePlus Real-Time PCR System (Applied Biosystems, Foster City, CA).

DNA Methylation Analyses. The reference sequence was derived from the National Center for Biotechnology Information sequence database (NC_000017.10). The CpG Island Searcher Program (Takai and Jones, 2003) was used to identify CGIs located within the $30 \mathrm{~kb}$ upstream of SLC47A1. A CGI was defined by the following criteria: GC > 50\%, observed CpG/expected CpG > 0.60, length > $200 \mathrm{bp}$, and gap between an adjacent island > $100 \mathrm{bp}$. Methylated DNA was enriched with the EpiXplore Methylated DNA Enrichment Kit (TakaRa) according to the manufacturer's protocol. We designed primers within each CGI to perform PCR with methylation-enriched DNA (Supplemental Table 2). Real-time PCR was performed as described above using SYBR Premix Ex Taq GC (TakaRa). We normalized the values of the methylation status in the CGIs using the primer designed within the CGI located upstream of the TSS of the CYP3A4 gene, which was previously shown to be hypermethylated in human liver samples (Hirota et al., 2004). A combined bisulfite restriction analysis was performed to evaluate the methylation status in some CGIs that were not amplified by real-time PCR. Isolated DNA was treated with sodium bisulfite using the EpiTect Bisulfite Kit (QIAGEN) and was amplified by PCR using bisulfite conversionbased primers (Supplemental Table 3). DNA amplification was performed with Epi Taq HS (Takara) under the following conditions: $30-37$ cycles at $98^{\circ} \mathrm{C}$ for 10 seconds, $50-60^{\circ} \mathrm{C}$ for 30 seconds, and $72^{\circ} \mathrm{C}$ for 30 seconds. PCR products were digested by specific restriction enzymes and separated by $3.0 \%$ agarose gel electrophoresis, and the intensity of the bands was quantified with a fluorescence image analyzer (Hitachi, Tokyo, Japan).

Demethylation by 5-Aza-2'-Deoxycytidine. All cells were treated with $5 \mu \mathrm{M}$ 5-aza-2'-deoxycytidine (5-aza-dC; Sigma-Aldrich) and dimethylsulfoxide ( $0.1 \%$, final concentration) as the vehicle control for 72 hours (Saito et al., 2013). Cells were dissociated enzymatically using TrypLE Express (Invitrogen), washed, and resuspended in phosphate-buffered saline.

Semiquantitative RT-PCR. Total RNA isolation and cDNA synthesis was performed as described above. cDNA amplification was conducted using primers specific for MATE1 (NM_018242) and GAPDH (NM_002046). Primers specific for MATE1 cDNA amplification were 5'-ATGCTGTTTCCCACCTCTTTG-3' (forward) and 5'CCGAGGCACGTTGTTTACTT-3' (reverse). Primers specific for GAPDH amplification were 5' - ATCAAGAAGGTGGTGAAGCAG-3' (forward) and 5'-TCGCTGTTGAAGTCAGAGGAG-3' (reverse). The amplification of GAPDH cDNA served as an internal control. PCR amplification for MATE1 and GAPDH was performed with KOD SYBR quantitative polymerase chain reaction (qPCR) mix (Toyobo, Osaka, Japan) under the following conditions: $98^{\circ} \mathrm{C}$ for 2 minutes and 38 cycles at $98^{\circ} \mathrm{C}$ for 10 seconds, $60^{\circ} \mathrm{C}$ for 10 seconds, and $68^{\circ} \mathrm{C}$ for 30 seconds. PCR amplification was also performed with AmpliTaq Gold DNA polymerase (Applied Biosystems) under the following conditions: $95^{\circ} \mathrm{C}$ for 9 minutes and 30 cycles at $95^{\circ} \mathrm{C}$ for 30 seconds, 
$55^{\circ} \mathrm{C}$ for 30 seconds, $72^{\circ} \mathrm{C}$ for 30 seconds, and $72^{\circ} \mathrm{C}$ for 5 minutes, respectively. PCR products were resolved on a $3 \%$ agarose gel and stained with ethidium bromide.

Construction of Reporter Plasmids. We attempted to investigate the role of CGIs $(-27,176$ to $-25,203 \mathrm{bp})$ on the transcriptional activity of the $S L C 47 A 1$ promoter, and we generated a fragment of the SLC47A1 promoter and fragments of CGIs of various lengths by nested-PCR assays (primer sets are described in Supplemental Table 4). Each amplified fragment of the CGI was digested and inserted into the pGL4.10 basic vector (Promega) between the KpnI and EcoRV sites. The fragment of the SLC47A1 promoter was also digested with NcoI and HindIII and inserted into the pGL4.10 basic vector or joined to the $3^{\prime}$ end of the CGIs.

Cell Transfection and Luciferase Assays. HepG2 cells were plated on a 24 -well plate at a density of $1.0 \times 10^{5}$ cells/well and were transfected with reporter vectors using Lipofectamine 2000 (Invitrogen) following the manufacturer's instructions. Luciferase activity was measured by the Dual-Luciferase Reporter Assay System (Promega).

Chromosome Conformation Capture Assay. A chromosome conformation capture (3C) assay was performed as previously described (Hagège et al., 2007). HepG2 cells were crosslinked with $1 \%$ formaldehyde, lysed, and digested overnight with the PstI restriction enzyme (TaKaRa) to obtain the 3C template. Samples were reverse-crosslinked at $65^{\circ} \mathrm{C}$ overnight, and DNA was purified by phenol-chloroform extraction and ethanol precipitation. Generation of the control 3C template using bacterial artificial chromosome DNA clones was essentially the same as described above. Bacterial artificial chromosome clone RP11-191D16 (Advanced Geno Techs Co., Tsukuba, Japan) containing the MATE1 gene locus was used for the assay. To control differences in the amount of DNA in different samples, an internal control was used within two PstI sites in the beta-actin locus. Quantification of the ligation products was performed using THUNDERBIRD Probe qPCR Mix (Toyobo) with the appropriate primers and the TaqMan probe designed at an anchor fragment including the downstream region of the CGI ( $-25,719$ to $-24,202 \mathrm{bp})$ using $3 \mathrm{C}$ templates and the control template. Sequences of the primers and the TaqMan probe used in the 3C assay are listed in Supplemental Table 5.

Deletion of CGIs Using the Clustered Regularly Interspaced Short Palindromic Repeats-Associated RNA-Guided Endonuclease Cas9 System. To generate the vector encoding a single guide RNA (sgRNA) and puromycin resistance, the pGL3-U6sgRNA-PGK-puromycin vector (ID 51133) was purchased from Addgene (Cambridge, MA). A clustered regularly interspaced short palindromic repeats (CRISPR)-associated RNA-guided endonuclease Cas9 system kit (Guide-it CRISPR/Cas 9; TaKaRa) was also used to generate the vector encoding an sgRNA and Cas9. We designed guide RNAs using an online tool (http://crispr.dbcls.jp). DNA oligonucleotides harboring variable 20 -nt sequences for Cas 9 targeting were annealed to generate short double-strand DNA fragments with 4-bp overhangs compatible with ligation into the BsaI site of the pGL3-U6-sgRNA-PGKpuromycin vector (Addgene) or the pGuide-it CRISPR/Cas9 vector (TaKaRa). HepG2 cells were plated on a six-well plate at a density of $5.0 \times 10^{5}$ cells/well and were transfected using Lipofectamine 3000 (Invitrogen) with $2.5 \mu \mathrm{g}$ of each vector. To select transfected cells, $2 \mu \mathrm{g} / \mathrm{ml}$ puromycin was added to the cell growth medium for 2 days. Cells were cloned by limiting dilutions, and DNA was extracted from the cloned cells for PCR amplification to analyze the deleted region by the CRISPR/Cas9 system (Supplemental Fig. 1A). MATE1 mRNA expression in the established clones was evaluated using a qPCR analysis. Sequences of the oligos and primers are described in Supplemental Table 6 .

Statistical Analysis. Data from at least three independent experiments are expressed as the mean \pm S.E.M. The means for two groups were compared with an unpaired $t$ test (two-tailed). Comparisons of means for multiple groups against controls were analyzed with Tukey-Kramer's multiple-comparisons test. A 5\% level of probability was considered significant. Relationship correlations between variables were assessed using Spearman's rank method.

\section{Results}

Quantification of MATE1 mRNA and a DNA Methylation Analysis of CGIs. Twelve CGIs were detected within the $30 \mathrm{~kb}$ upstream of $S L C 47 A 1$ (Fig. 1A). CGI-12 contained the TSS of the SLC47A1 gene. The relationship between hepatic MATE1 expression and the DNA methylation status in CGIs was analyzed in human liver samples. The methylation status of CGIs was examined by an affinity-based methylation capture assay or combined bisulfite restriction analysis. Approximately 20 -fold interindividual variability in MATE1 mRNA expression levels was observed in liver samples, and mRNA expression levels were negatively correlated with the methylation levels of CGI-3 ( $-27,176$ to $-25,203 \mathrm{bp}$ ) (Fig. 1B), whereas no correlation was found in CGI-12 containing the TSS. These results suggest that the DNA methylation of CGI-3 may play an important role in interindividual differences in hepatic MATE1 expression.

Evaluation of MATE1 mRNA Expression in Demethylating Agent-Treated Cells. To analyze the contribution of DNA methylation to MATE1 expression in cells with positive or negative MATE1 expression, four cell lines were exposed to the DNA methyltransferase inhibitor 5-aza-dC. MATE1 mRNA expression levels remained unchanged after treatment with 5-aza-dC in MATE1-positive HepG2 and HEK293 cells, whereas a marked increase in MATE1 mRNA expression levels was noted in MATE1-negative LS174T and HaCaT cells (Fig. 2).

The Effect of CGIs on Transcriptional Activity of the SLC47A1 Promoter. CGI-3 showed that DNA methylation status correlated with hepatic MATE1 expression and was located $27 \mathrm{~kb}$ from the TSS. We evaluated the enhancer activity of CGI-3 using luciferase vectors containing the $S L C 47 A 1$ promoter region. To identify important regions for transcriptional activity, SLC47A1 promoter vectors containing various segments of CGI-3 were used (Fig. 3). Constructs F1, F2, F3, and F4 had significantly higher transcriptional activities than the SLC47A1 promoter-only construct. In contrast, transcriptional activity of construct F5 was not significantly different from that of the SLC47A1 promoteronly construct. These results indicate that the deleted region between constructs F4 and F5 ( $-25,670$ to $-25,246 \mathrm{bp})$ played an important role in the enhancer activity of CGI-3.

Analysis of Long-Range DNA Interactions between the SLC47A1 Gene Locus and the CGI. It is increasingly clear that genomic regulation by distal regulatory regions is associated with direct interactions between distal regulatory regions and target gene promoters by DNA loop formation (Vakoc et al., 2005; Mimura et al., 2012; Lee et al., 2015). We performed a $3 \mathrm{C}$ assay to analyze the proximity of the downstream region within CGI-3 (referred to as 3' CGI-3) with the SLC47A1 gene locus in MATE1-positive HepG2 cells. We used a PCR primer located in the PstI fragment that contains 3' CGI-3 and paired it with primers in restriction fragments throughout a $30-\mathrm{kb}$ region (primer sequences available in Supplemental Table 5). We found that the promoter fragment interacted most strongly with nearby restriction fragments and that interaction frequencies markedly decreased for fragments located farther away, whereas the frequency in the proximal region of the TSS was similar to that in the region immediately adjacent to CGI-3 (Fig. 4). This result suggests long-range DNA looping between the SLC47A1 gene and CGI-3 in HepG2 cells. 

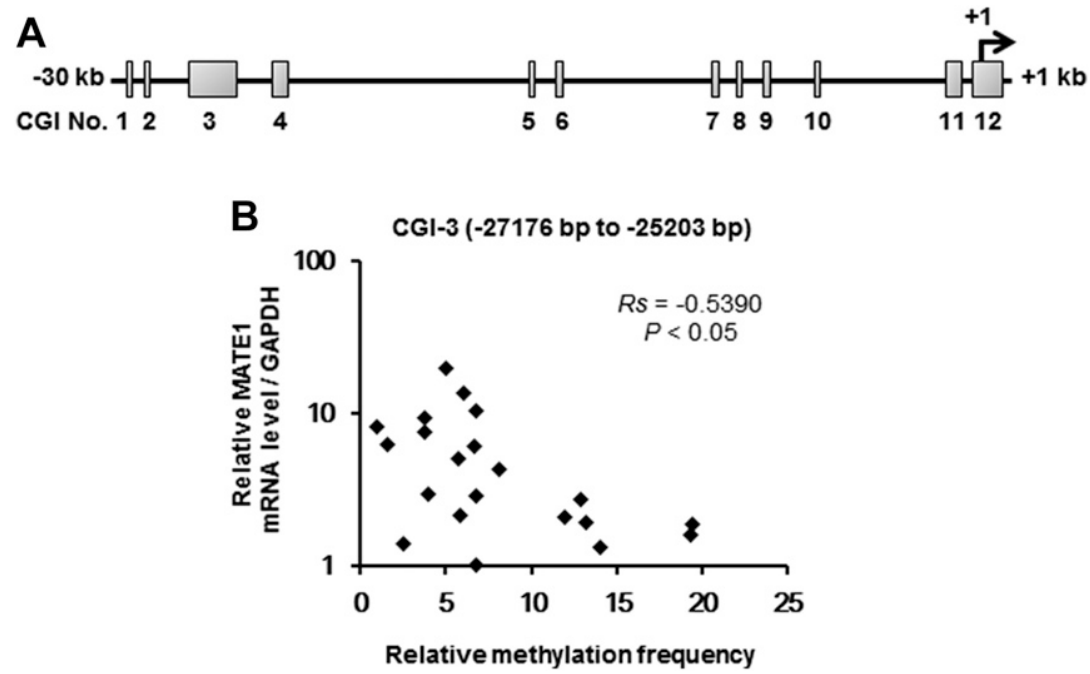

Fig. 1. Correlation analysis between MATE1 mRNA levels and methylation frequencies of the CGI in Caucasian liver samples. (A) Schematic of the upstream region of the SLC47A1 gene from $30 \mathrm{~kb}$ upstream to $1 \mathrm{~kb}$ downstream. +1 indicates the TSS of SLC47A1. Gray boxes show CGIs and are CGI-1 to CGI-12. (B) Correlation between the mRNA expression levels of MATE1 and methylation levels of CGI-3. Rs, Spearman's rank-order correlation analysis.

The Contribution of CGIs to MATE1 Expression in HepG2 Cells. The results obtained above show the importance of 3' CGI-3 including $-25,670$ to $-25,246$ bp for hepatic MATE1 expression. To further understand the role of CGI-3 in MATE1 expression, we deleted the region surrounding 3' CGI-3 in HepG2 cells using the CRISPR/Cas9 system. In brief, the paired synthesized oligonucleotides (Supplemental Table 6) for sgRNA targeting the region were annealed and cloned into the pGL3-U6-sgRNA-PGK-puromycin vector. In accordance with the manufacturer's instructions, HepG2 cells were transfected with sgRNA with Cas9 plasmid by the lipofection method. 3' CGI-3-deleted clones and 3' CGI-3-not deleted clones were obtained (referred to as deletion clones and control clones, respectively) (Supplemental Fig. 1). We confirmed the deletion of 3' CGI-3 by the direct sequencing method (Supplemental Fig. 2). MATE1 mRNA expression levels were significantly lower in deletion clones than in control clones (Fig. 5). This result indicates that the region surrounding 3' CGI-3 increases $S L C 47 A 1$ transcription in HepG2 cells.

\section{Discussion}

The expression level of Mate1 in rats has been associated with tubular secretion and blood levels of the substrate drug (Nishihara et al., 2007). Interindividual differences in MATE1 expression play an important role in the altered function of MATE1. Some genetic variations in the SLC47A1 promoter region were evaluated and were shown to affect $S L C 47 A 1$ promoter activity by altering binding of some transcription factors (Kajiwara et al., 2007; Ha Choi et al., 2009; Kim et al., 2013); however, their contribution was not large enough to explain the interindividual variability in hepatic MATE1 expression. Most studies that focused on the relationship between DNA methylation and gene expression only analyzed the methylation status of CGIs located near target gene promoters. However, CGIs far from the target gene locus have been shown to affect gene regulation (Yoon et al., 2002, 2005). The relationship between epigenetic regulation and SLC47A1 currently remains unclear; therefore, we performed a comprehensive DNA methylation analysis in this study to precisely evaluate the role of DNA methylation in interindividual differences in hepatic MATE1 expression. Before the methylation analysis, we analyzed the polymorphism in the promoter region of the $S L C 47 A 1$ gene (rs72466470). The variant was not identified in the promoter region. In the methylation analysis, MATE1 mRNA expression levels negatively correlated with methylation levels of CGIs located in the 27-kb upstream region of the $S L C 47 A 1$ gene. A number of genes including drug transporters were revealed to be suppressed by promoter DNA methylation in cancer cell lines (Schaeffeler et al., 2011; Tang et al., 2011; Ikehata et al., 2012). DNA methylation in gene promoters is crucial for gene transcription. However, a relationship was not found between DNA methylation in the SLC47A1 promoter and MATE1 mRNA expression. This result suggested that DNA methylation status in the SLC47A1 promoter is not important for interindividual variability in MATE1 expression.

To analyze the role of DNA methylation in MATE1 expression, the effects of demethylation on MATE1 expression were evaluated using four cell lines. MATE1-negative cell lines showed an increase in MATE1 mRNA expression by demethylation, whereas MATE1-positive cell lines did not. Tissuespecific DNA methylation was reported previously in mammals (Aoki et al., 2008; Imai et al., 2013), and demethylating agents only increased the expression of organic cation/ carnitine transporter 2 mRNA in the human tissue cell lines
Cell lines

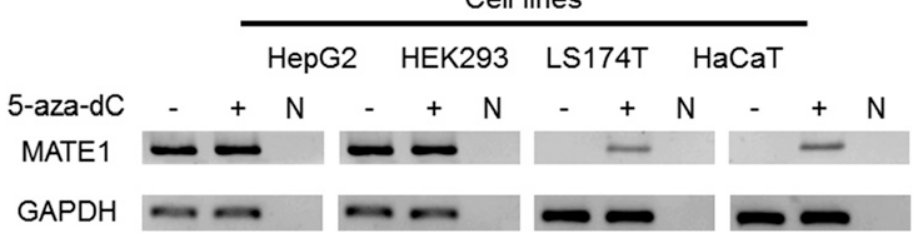

Fig. 2. Effects of 5-aza-dC on MATE1 mRNA expression in four cell lines. All cell lines were treated with 5-aza-dC for 72 hours before harvesting. MATE1 mRNA expression was assessed using RT-PCR. N, no template control. 


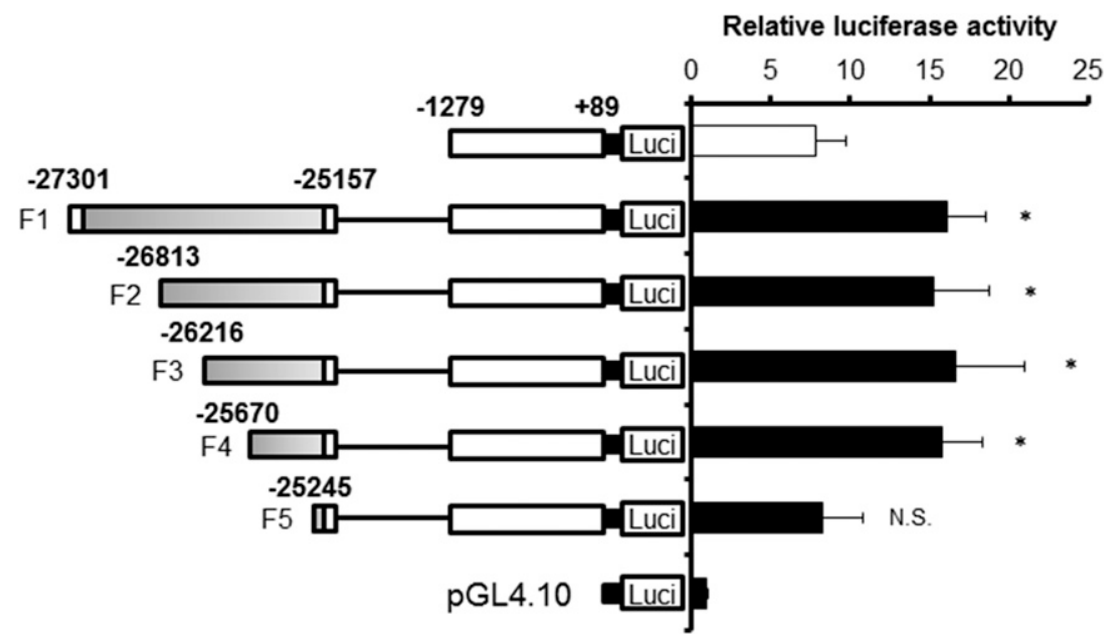

Fig. 3. Luciferase activities in the SLC47A1 promoter and CGI-3. Luciferase (Luci) reporter gene constructs were transiently transfected into HepG2 cells. Gray and white boxes show CGI-3 ( $-27,176$ to $-25,203 \mathrm{bp})$ and the $S L C 47 A 1$ promoter region, respectively. Luciferase values were normalized to the pGL4.70 vector and the mean value obtained with the pGL4.10 vector as $100 \%$. Data represent the mean \pm S.D. $(n=3)$ and were analyzed using Dunnett's test. ${ }^{*} P<0.05$ (significantly different from the control vector containing only the SLC47A1 gene promoter region). N.S., not significant.

showing methylated DNA status ( $Q u$ et al., 2013). These findings suggest that methylated DNA in MATE1-negative cell lines contributes to control the expression of MATE1.

The luciferase reporter assay showed that the region from 25,670 to $-25,246$ bp in CGI-3 was important for increasing the transcriptional activity of the $S L C 47 A 1$ promoter. In addition, HepG2 cells that showed the complete absence of the region surrounding $3^{\prime}$ CGI-3 had significantly lower expression levels of MATE1 mRNA. These results suggest the importance of this region for $S L C 47 A 1$ gene regulation in HepG2 cells. The relationship between DNA methylation status in CGI shores and gene expression was described previously (Ji et al., 2010). CGI shores are regions of DNA with a low density of $\mathrm{CpG}$ dinucleotides that are located near CGIs. Previous studies revealed that tissue-specific DNA methylation and cancer-related methylation changes occurred in CGI shores (Doi et al., 2009; Irizarry et al., 2009). Since the region from $-25,670$ to $-25,246 \mathrm{bp}$ is located near the $3^{\prime}$ end of CGI-3 and shows a low density of $\mathrm{CpG}$, it may fall under the category of a CGI shore and may play an important role in SLC47A1 gene regulation.

Recent studies revealed that DNA-DNA interactions between enhancer elements and target genes are necessary for the function of enhancers that are distant from their target genes (Hwang et al., 2013; Wang et al., 2015). Based on these findings, we analyzed DNA interactions between the SLC47A1 gene locus and CGI. The results of the 3C assay revealed the spatial proximity of 3 ' CGI-3 to the proximal region of the SLC47A1 TSS caused by DNA looping of the $S L C 47 A 1$ locus in HepG2 cells. These results suggest that this region acts as an enhancer and regulates the $S L C 47 A 1$ gene in HepG2 cells.

In this study, we showed that the SLC47A1 gene was regulated through the CGI located in the $27 \mathrm{~kb}$ upstream of the SLC47A1 gene and that interindividual variability in hepatic MATE1 expression correlated with DNA methylation in this CGI. The contribution of altered MATE1 mRNA expression to protein expression and in vivo activity was not elucidated in this study. Further studies are needed to clarify whether DNA methylation status in CGIs is involved in the protein expression in the liver and in the pharmacokinetics of substrate drugs of MATE1. The mechanisms responsible for variability in DNA methylation status in CGIs and for regulation of the SLC47A1 gene via DNA methylation currently remain unclear. It is well known that environmental factors, including diet, long-term diseases, and drug exposure, can provoke epigenetic responses (Jaenisch and Bird, 2003). In addition to the evaluation of DNA methylation, analysis of the

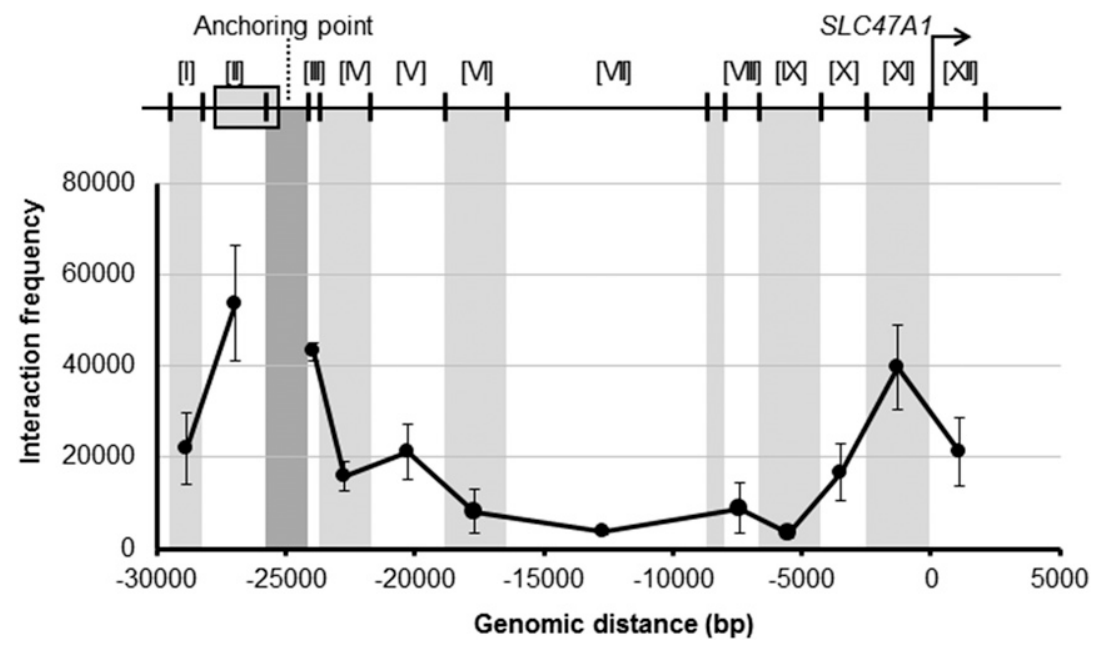

Fig. 4. $3 \mathrm{C}$ assay of the $S L C 47 A 1$ gene locus in HepG2 cells. The vertical lines show the positions of PstI restriction sites and the gray box shows CGI-3. The constant primer and TaqMan probe were designed in the PstI fragment containing CGI-3 (anchoring point), and crosslinking frequencies between the anchor fragment and PstI fragment in the SLC47A1 gene promoter were analyzed by TaqMan real-time PCR. Data represent the mean \pm S.D. $(n=3)$. 


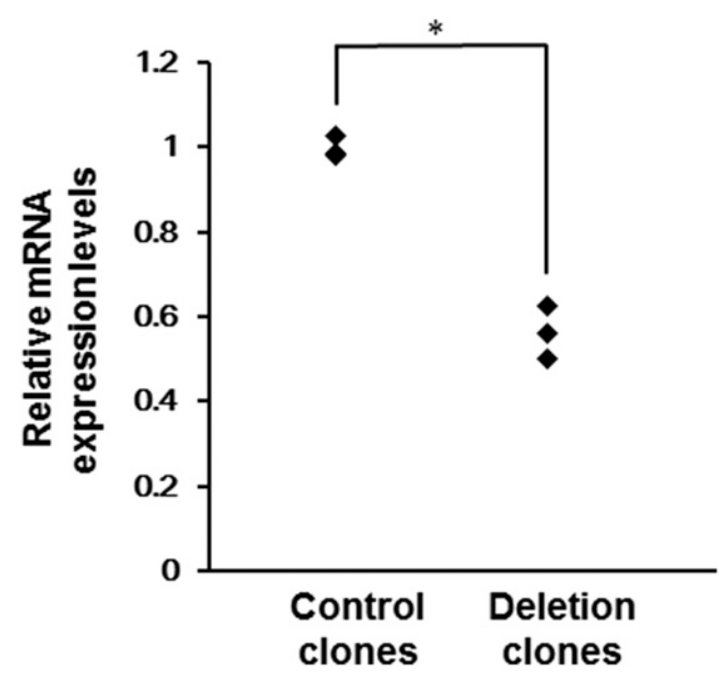

Fig. 5. Effects of the deletion of the CGI on MATE1 mRNA expression in HepG2 cells. MATE1 mRNA expression was measured by RT-qPCR. Results were normalized to the expression of GAPDH. ${ }^{*} P<0.05$ (statistically analyzed using the $t$ test).

environmental factors contributing to interindividual differences in MATE1 expression is important. Lin et al. (2013) reported previously that long-term exposure to cisplatin enhanced SLC22A1 promoter methylation by activating DNA methyltransferase 1 (DNMT1), but DNMT regulation of DNA methylation of the SLC47A1 gene was not identified. Investigation of the role of DNMT in the regulation of DNA methylation of the SLC47A1 gene is key to elucidating the mechanisms that cause interindividual variability in hepatic MATE1 expression.

\section{Acknowledgments}

The authors appreciate the technical assistance provided by the Research Support Center and Research Center for Human Disease Modeling at Kyushu University Graduate School of Medical Sciences.

\section{Authorship Contributions}

Participated in research design: Tanaka, Hirota, Ieiri.

Conducted experiments: Tanaka, Hirota.

Performed data analysis: Tanaka, Hirota.

Wrote or contributed to the writing of the manuscript: Tanaka, Hirota, Ieiri.

\section{References}

Aoki M, Terada T, Kajiwara M, Ogasawara K, Ikai I, Ogawa O, Katsura T, and Inui K (2008) Kidney-specific expression of human organic cation transporter 2 (OCT2/SLC22A2) is regulated by DNA methylation. Am J Physiol Renal Physiol 295:F165-F170.

Bird A (2002) DNA methylation patterns and epigenetic memory. Genes Dev 16:6-21. Bram EE, Stark M, Raz S, and Assaraf YG (2009) Chemotherapeutic drug-induced ABCG2 promoter demethylation as a novel mechanism of acquired multidrug resistance. Neoplasia 11:1359-1370.

Christensen MMH, Brasch-Andersen C, Green H, Nielsen F, Damkier P, BeckNielsen H, and Brosen K (2011) The pharmacogenetics of metformin and its impact on plasma metformin steady-state levels and glycosylated hemoglobin A1c. Pharmacogenet Genomics 21:837-850.

Deaton AM and Bird A (2011) CpG islands and the regulation of transcription. Genes Dev 25:1010-1022.

Doi A, Park I-H, Wen B, Murakami P, Aryee MJ, Irizarry R, Herb B, Ladd-Acosta C Rho J, Loewer S, et al. (2009) Differential methylation of tissue- and cancer-specific $\mathrm{CpG}$ island shores distinguishes human induced pluripotent stem cells, embryonic stem cells and fibroblasts. Nat Genet 41:1350-1353.

Ha Choi J, Wah Yee S, Kim MJ, Nguyen L, Ho Lee J, Kang JO, Hesselson S, Castro RA, Stryke D, Johns SJ, et al. (2009) Identification and characterization of novel polymorphisms in the basal promoter of the human transporter, MATE1. Pharmacogenet Genomics 19:770-780.
Hagège H, Klous P, Braem C, Splinter E, Dekker J, Cathala G, de Laat W, and Forné $\mathrm{T}$ (2007) Quantitative analysis of chromosome conformation capture assays (3CqPCR). Nat Protoc 2:1722-1733.

Hirota T, Ieiri I, Takane H, Maegawa S, Hosokawa M, Kobayashi K, Chiba K, Nanba E, Oshimura M, Sato T, et al. (2004) Allelic expression imbalance of the human CYP3A4 gene and individual phenotypic status. Hum Mol Genet 13:2959-2969.

Hwang YC, Zheng Q, Gregory BD, and Wang LS (2013) High-throughput identification of long-range regulatory elements and their target promoters in the human genome. Nucleic Acids Res 41:4835-4846.

Ikehata M, Ueda K, and Iwakawa S (2012) Different involvement of DNA methylation and histone deacetylation in the expression of solute-carrier transporters in 4 colon cancer cell lines. Biol Pharm Bull 35:301-307.

Imai S, Kikuchi R, Kusuhara H, and Sugiyama Y (2013) DNA methylation and histone modification profiles of mouse organic anion transporting polypeptides. Drug Metab Dispos 41:72-78.

Irizarry RA, Ladd-Acosta C, Wen B, Wu Z, Montano C, Onyango P, Cui H, Gabo K, Rongione M, Webster M, et al. (2009) The human colon cancer methylome shows similar hypo- and hypermethylation at conserved tissue-specific $\mathrm{CpG}$ island shores. Nat Genet 41:178-186.

Jaenisch R and Bird A (2003) Epigenetic regulation of gene expression: how the genome integrates intrinsic and environmental signals. Nat Genet 33 (Suppl): $245-254$

Ji H, Ehrlich LIR, Seita J, Murakami P, Doi A, Lindau P, Lee H, Aryee MJ, Irizarry RA, Kim K, et al. (2010) Comprehensive methylome map of lineage commitment from haematopoietic progenitors. Nature 467:338-342.

Kajiwara M, Terada T, Asaka J, Ogasawara K, Katsura T, Ogawa O, Fukatsu A, Doi $\mathrm{T}$, and Inui K (2007) Critical roles of Sp1 in gene expression of human and rat H+ lorganic cation antiporter MATE1. Am J Physiol Renal Physiol 293:F1564-F1570.

Kim TH, Kim KH, Park HJ, Kim S, and Choi JH (2013) Identification and functional characterization of novel MATE1 genetic variations in Koreans. Biochem Biophys Res Commun 434:334-340.

Lee K, Hsiung CC, Huang P, Raj A, and Blobel GA (2015) Dynamic enhancer-gene body contacts during transcription elongation. Genes Dev 29:1992-1997.

Lin R, Li X, Li J, Zhang L, Xu F, Chu Y, and Li J (2013) Long-term cisplatin exposure promotes methylation of the OCT1 gene in human esophageal cancer cells. Dig Dis Sci 58:694-698.

Masuda S, Terada T, Yonezawa A, Tanihara Y, Kishimoto K, Katsura T, Ogawa O, and Inui $\mathrm{K}$ (2006) Identification and functional characterization of a new human kidney-specific $\mathrm{H}+$ /organic cation antiporter, kidney-specific multidrug and toxin extrusion 2. J Am Soc Nephrol 17:2127-2135.

Mimura I, Nangaku M, Kanki Y, Tsutsumi S, Inoue T, Kohro T, Yamamoto S, Fujita T, Shimamura T, Suehiro J, et al. (2012) Dynamic change of chromatin conformation in response to hypoxia enhances the expression of GLUT3 (SLC2A3) by cooperative interaction of hypoxia-inducible factor 1 and KDM3A. Mol Cell Biol 32: 3018-3032.

Moon HH, Kim SH, and Ku JL (2016) Correlation between the promoter methylation status of ATP-binding cassette sub-family G member 2 and drug sensitivity in colorectal cancer cell lines. Oncol Rep 35:298-306.

Niemi M, Kivistö KT, Hofmann U, Schwab M, Eichelbaum M, and Fromm MF (2005) Fexofenadine pharmacokinetics are associated with a polymorphism of the SLCO1B1 gene (encoding OATP1B1). Br J Clin Pharmacol 59:602-604.

Nishihara K, Masuda S, Ji L, Katsura T, and Inui K (2007) Pharmacokinetic significance of luminal multidrug and toxin extrusion 1 in chronic renal failure rats. Biochem Pharmacol 73:1482-1490.

Otsuka M, Matsumoto T, Morimoto R, Arioka S, Omote H, and Moriyama Y (2005) A human transporter protein that mediates the final excretion step for toxic organic cations. Proc Natl Acad Sci USA 102:17923-17928.

Qu Q, Qu J, Zhan M, Wu LX, Zhang YW, Lou XY, Fu LJ, and Zhou HH (2013) Different involvement of promoter methylation in the expression of organic cation/carnitine transporter 2 (OCTN2) in cancer cell lines. PLoS One 8:e76474.

Saito J, Hirota T, Furuta S, Kobayashi D, Takane H, and Ieiri I (2013) Association between DNA methylation in the miR-328 5 '-flanking region and inter-individual differences in miR-328 and BCRP expression in human placenta. PLoS One 8: e72906.

Saxonov S, Berg P, and Brutlag DL (2006) A genome-wide analysis of CpG dinucleotides in the human genome distinguishes two distinct classes of promoters. Proc Natl Acad Sci USA 103:1412-1417.

Schaeffeler E, Hellerbrand C, Nies AT, Winter S, Kruck S, Hofmann U, van der Kuip H, Zanger UM, Koepsell H, and Schwab M (2011) DNA methylation is associated with downregulation of the organic cation transporter OCT1 (SLC22A1) in human hepatocellular carcinoma. Genome Med 3:82.

Stocker SL, Morrissey KM, Yee SW, Castro RA, Xu L, Dahlin A, Ramirez AH, Roden DM, Wilke RA, McCarty CA, et al. (2013) The effect of novel promoter variants in MATE1 and MATE2 on the pharmacokinetics and pharmacodynamics of metformin. Clin Pharmacol Ther 93:186-194.

Takai D and Jones PA (2003) The CpG island searcher: a new WWW resource. In Silico Biol 3:235-240.

Tang SC, Wu MF, Wong RH, Liu YF, Tang LC, Lai CH, Hsu CP, and Ko JL (2011) Epigenetic mechanisms for silencing glutathione S-transferase $\mathrm{m} 2$ expression by hypermethylated specificity protein 1 binding in lung cancer. Cancer 117: 3209-3221.

To KKW, Zhan Z, and Bates SE (2006) Aberrant promoter methylation of the ABCG2 gene in renal carcinoma. Mol Cell Biol 26:8572-8585.

Toyama K, Yonezawa A, Masuda S, Osawa R, Hosokawa M, Fujimoto S, Inagaki N, Inui $\mathrm{K}$, and Katsura T (2012) Loss of multidrug and toxin extrusion 1 (MATE1) is associated with metformin-induced lactic acidosis. Br J Pharmacol 166:1183-1191.

Tzvetkov MV, Vormfelde SV, Balen D, Meineke I, Schmidt T, Sehrt D, Sabolić I, Koepsell H, and Brockmöller J (2009) The effects of genetic polymorphisms in the organic cation transporters OCT1, OCT2, and OCT3 on the renal clearance of metformin. Clin Pharmacol Ther 86:299-306. 
Vakoc CR, Letting DL, Gheldof N, Sawado T, Bender MA, Groudine M, Weiss MJ, Dekker J, and Blobel GA (2005) Proximity among distant regulatory elements at the $\beta$-globin locus requires GATA-1 and FOG-1. Mol Cell 17:453-462.

Wang D, Papp AC, and Sun X (2015) Functional characterization of CYP2D6 enhancer polymorphisms. Hum Mol Genet 24:1556-1562.

Warton K and Samimi G (2015) Methylation of cell-free circulating DNA in the diagnosis of cancer. Front Mol Biosci 2:13.

Wu LX, Wen CJ, Li Y, Zhang X, Shao YY, Yang Z, and Zhou HH (2015) Interindividual epigenetic variation in ABCB1 promoter and its relationship with ABCB1 expression and function in healthy Chinese subjects. $\mathrm{Br}$ J Clin Pharmacol 80:1109-1121.

Yamasaki Y, Ieiri I, Kusuhara H, Sasaki T, Kimura M, Tabuchi H, Ando Y, Irie S, Ware J, Nakai Y, et al. (2008) Pharmacogenetic characterization of sulfasalazine disposition based on NAT2 and ABCG2 (BCRP) gene polymorphisms in humans. Clin Pharmacol Ther 84:95-103.
Yasar U, Greenblatt DJ, Guillemette C, and Court MH (2013) Evidence for regulation of UDP-glucuronosyltransferase (UGT) 1A1 protein expression and activity via DNA methylation in healthy human livers. J Pharm Pharmacol 65:874-883.

Yoon B, Herman H, Hu B, Park YJ, Lindroth A, Bell A, West AG, Chang Y, Stablewski A, Piel JC, et al. (2005) Rasgrf1 imprinting is regulated by a CTCFdependent methylation-sensitive enhancer blocker. Mol Cell Biol 25:11184-11190. Yoon BJ, Herman H, Sikora A, Smith LT, Plass C, and Soloway PD (2002) Regulation of DNA methylation of Rasgrf1. Nat Genet 30:92-96.

Address correspondence to: Dr. Ichiro Ieiri, Division of Clinical Pharmacy, Department of Clinical Pharmacokinetics, Graduate School of Pharmaceutical Sciences, Kyushu University, Fukuoka 8128582, Japan. E-mail: ieiri-ttr@ umin.ac.jp 